\title{
MONTE CARLO SIMULATION TO DETERMINE CONDITIONS FOR OPTICAL MOLECULAR IMAGING OF VASCULAR DISEASE
}

\author{
Mambidzeni Madzivire, Christopher Riederer, James Greenleaf \\ Mayo Clinic and Foundation, 200 First Street SW, Rochester, MN 55905
}

\begin{abstract}
Atherosclerosis is a significant contributor to cardiovascular disease, which is a leading cause of death in the United States. Optical imaging is emerging as a promising molecular imaging tool to detect the biomarkers of vascular disease and plaque vulnerability. We describe a Monte Carlo method of investigating the use of targeted fluorescent nanoparticles as a reporter agent for optical molecular imaging. In particular, we used the model to provide insight into the optimum excitation and emission wavelengths for in vivo imaging in the presence of blood and autofluorescence.
\end{abstract}

Index Terms - Monte Carlo, molecular imaging, atherosclerosis, quantum dots, optical imaging

\section{INTRODUCTION}

Atherosclerosis is a significant contributor to cardiovascular disease, which is a leading cause of death in the United States [1]. Vulnerable atherosclerotic plaques are of particular concern because they can rupture spontaneously, causing thrombosis which could occlude blood flow to major organs such as the heart and brain. These types of plaque are difficult to detect based on their morphology, since they are not necessarily occlusive, and there is currently no established clinical method to detect them before they rupture. There is active research aimed at detecting the various molecular markers of vascular inflammation and plaque vulnerability using targeted contrast agents in ultrasound, MRI, CT and PET [2, 3].

Optical molecular imaging is emerging as a promising tool for in vivo molecular imaging of vulnerable plaque. Our approach is to use commercially available fluorescent semiconductor nanoparticles (Molecular Probes, Invitrogen) that are covalently linked to an antibody to the biomarker of interest as the reporter agent. Semiconductor nanoparticles (quantum dots) offer several unique optical properties including high luminescence, size tunable emission frequencies, wide absorption and narrow emission spectra [4, 5]. However, the absorption of light by blood and tissue limits the ability to fully exploit all wavelengths of light for excitation and emission.
Previous research by several groups indicates that an "optical window" between $600-900 \mathrm{~nm}$ exists for which the absorption due to blood, as well as tissue autofluorescence, is minimized [6]. While there have been extensive investigations of the propagation of photons in tissue, including fluorescence [7-9], there are none that have specifically sought to understand how the excitation and emission wavelengths can be optimized for in vivo vascular imaging. We have designed a Monte Carlo program for further analysis of the optimal conditions for in vivo optical molecular imaging of quantum dots in the presence of blood.

\section{MONTE CARLO MODEL}

\subsection{General Model}

Monte Carlo simulations have been widely used to investigate the propagation of light in tissue. Our model is based on that by Wang, et al. [10], with modifications made in order to enable the generation and propagation of fluorescent photons at a second wavelength.

Briefly, photons are launched into the tissue and are advanced using step sizes and scattering angles that are dependent on the optical properties at the excitation wavelength. If a portion of the photon packet is absorbed in a layer containing fluorescent molecules, a decision is made whether that photon packet will be re-emitted as fluorescence based on the quantum yield. In the case where fluorescence occurs, that photon packet will be advanced using the optical properties corresponding to the emission wavelength. We assume that the initial photon packet can generate more than one fluorescent photon packet. However, fluorescent photon packets cannot generate additional fluorescence.

\subsection{Model Geometry}

The coronary artery consists of four layers: the endothelium, consisting of a thin layer of cells; the intima, consisting of connective tissue and some smooth muscle cells; the media, consisting mainly of smooth muscle cells, collagen and elastin; the adventitia, consisting of connective tissue. 
The geometrical model used in the Monte Carlo simulations is shown in Figure 1. It consists of five layers representing blood, quantum dots, intima, media and adventitia. The thickness of the blood was assumed to be the radius of a typical coronary artery and the thickness of the layers of the arterial wall were obtained from the literature.

For simplicity, the endothelial layer was not included in the simulations and the quantum dots are modeled as a separate layer between the blood and the intima.

\subsection{Model Parameters}

The parameters that were input into the algorithm included layer thickness $(d)$, absorption coefficient $\left(\mu_{\mathrm{a}}\right)$, scattering coefficient $\left(\mu_{\mathrm{s}}\right)$, refractive index $(\mathrm{n})$, fluorescence coefficient $\left(\mu_{\mathrm{f}}\right)$, mean cosine of scattering angle $(\mathrm{g})$ and quantum yield $(\phi)$. These values were estimated from the literature and from information provided through verbal communications with Invitrogen $[11,12]$ and are enumerated in Table 1. The top and bottom ambient layers were index matched to the adjacent tissue.

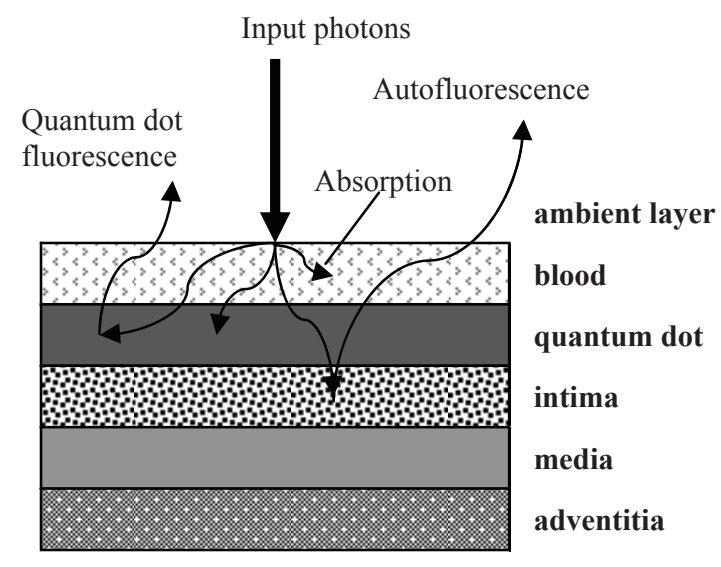

ambient layer

Figure 1. Geometry used for Monte Carlo model.

\begin{tabular}{|c|c|c|c|c|c|c|c|c|}
\hline & $\begin{array}{c}\lambda \\
{[\mathrm{nm}]}\end{array}$ & $\begin{array}{c}\text { Ambient } \\
\text { layer }\end{array}$ & Blood & Quantum Dot & Intima & Media & Adventitia & $\begin{array}{c}\text { Ambient } \\
\text { layer }\end{array}$ \\
\hline $\mathrm{d}[\mathrm{cm}]$ & & - & 0.170 & 0.010 & 0.025 & 0.035 & 0.030 & - \\
\hline $\mathbf{n}$ & & 1.4 & 1.4 & 1.4 & 1.4 & 1.4 & 1.4 & 1.4 \\
\hline \multirow[t]{4}{*}{$\mu_{\mathrm{a}}\left[\mathrm{cm}^{-1}\right]$} & 550 & - & 20.0 & $3.914 \times 10^{6} \mathrm{M}^{-1}$ & 9.0 & 5.0 & 12.5 & - \\
\hline & 600 & - & 8.0 & $2.878 \times 10^{6} \mathrm{M}^{-1}$ & 4.0 & 3.0 & 7.0 & - \\
\hline & 650 & - & 3.0 & $1.151 \times 10^{6} \mathrm{M}^{-1}$ & 3.5 & 2.3 & 5.5 & - \\
\hline & 700 & - & 1.0 & $0.025 \times 10^{6} \mathrm{M}^{-1}$ & 3.0 & 1.9 & 5.0 & - \\
\hline \multirow{4}{*}{$\mu_{\mathrm{s}}\left[\mathrm{cm}^{-1}\right]$} & 550 & - & 350 & 10 & 240 & 400 & 270 & - \\
\hline & 600 & - & 325 & 10 & 220 & 360 & 250 & - \\
\hline & 650 & - & 320 & 10 & 290 & 325 & 230 & - \\
\hline & 700 & - & 310 & 10 & 170 & 300 & 210 & - \\
\hline \multirow[t]{3}{*}{$\mu_{\mathrm{f}}\left[\mathrm{cm}^{-1}\right]$} & 550 & - & 0.0015 & $3.914 \times 10^{6} \mathrm{M}^{-1}$ & 0.00075 & 0.0015 & 0.0009 & - \\
\hline & 600 & - & 0.00075 & $2.878 \times 10^{6} \mathrm{M}^{-1}$ & 0.00045 & 0.00075 & 0.00052 & - \\
\hline & 650 & - & 0.000375 & $1.151 \times 10^{6} \mathrm{M}^{-1}$ & 0.00025 & 0.000375 & 0.00025 & - \\
\hline \multirow[t]{4}{*}{ g } & 550 & - & 0.99 & 0.90 & 0.85 & 0.91 & 0.79 & - \\
\hline & 600 & - & 0.99 & 0.90 & 0.85 & 0.90 & 0.80 & - \\
\hline & 650 & - & 0.99 & 0.90 & 0.85 & 0.90 & 0.81 & - \\
\hline & 700 & - & 0.99 & 0.90 & 0.85 & 0.90 & 0.82 & - \\
\hline \multirow[t]{3}{*}{$\phi$} & 550 & - & 0.005 & 0.65 & 0.00075 & 0.00003 & 0.00007 & - \\
\hline & 600 & - & 0.00011 & 0.65 & 0.00011 & 0.00025 & 0.00007 & - \\
\hline & 650 & - & 0.00025 & 0.65 & 0.00007 & 0.000163 & 0.000045 & - \\
\hline
\end{tabular}

Table 1. Input parameters for Monte Carlo algorithm.

\section{METHODS}

The code for the Monte Carlo simulations was programmed in ANSI C and executed in the Unix operating system on a
Sun Enterprise computer. Simulations were performed to investigate the effect of excitation wavelength, emission wavelength and quantum dot concentration on the absorption by blood as well as the fluorescence output. In 
each case, 100000 photons were traced. The absorption of excitation photons by the blood, the tissue autofluorescence and quantum dot fluorescence were recorded.

\section{RESULTS}

Preliminary results from the Monte Carlo simulation show that modifying the excitation and emission wavelengths and the quantum dot concentration has an effect on the fluorescence output.

Figure 2 shows the results of changing the excitation wavelength on the absorption due to blood. Increasing the excitation wavelength decreases the absorption by blood. Figure 3 shows the results of increasing the excitation wavelength while maintaining the emission wavelength at $650 \mathrm{~nm}$. This simulation corresponds to commercially available quantum dots that emit at $655 \mathrm{~nm}$. Figure 4 shows the results of increasing the excitation wavelength for an emission wavelength of $700 \mathrm{~nm}$. This simulation represents commercially available quantum dots that emit at $705 \mathrm{~nm}$. In both cases, increasing the excitation wavelength effectively increases the number of photons that are reemitted as fluorescence. In addition, increasing the concentration of the quantum dots leads to an elevated amount of fluorescence.

\section{DISCUSSION}

The results of the Monte Carlo simulations show that increasing the excitation wavelength causes a decrease in the amount of absorption by blood. This allows a greater percentage of the excitation light to reach the quantum dots and the underlying layers of tissue.

The results also indicate that increasing the excitation wavelength increases the amount of fluorescence for both the quantum dots emitting at $655 \mathrm{~nm}$ and at $705 \mathrm{~nm}$, despite the fact that the extinction coefficient of the quantum dots at longer wavelengths is significantly reduced. This may be due to more excitation photons reaching the quantum dots at the longer wavelength. This suggests that the reduced absorption of the quantum dots at longer wavelengths of light can be effectively counterbalanced by allowing more light to propagate through the blood.

\section{CONCLUSIONS}

Preliminary results from the Monte Carlo simulation show that optical molecular imaging using targeted quantum dots in the presence of blood may be feasible. Future work will involve testing additional wavelengths in order to find the optimal combination of excitation and emission wavelengths. Furthermore, we will investigate the minimum concentration of quantum dots that will be necessary to achieve fluorescence above the autofluorescence of arterial wall tissue. The Monte Carlo simulation could also provide guidelines regarding the required sensitivity of the appropriate detection system.

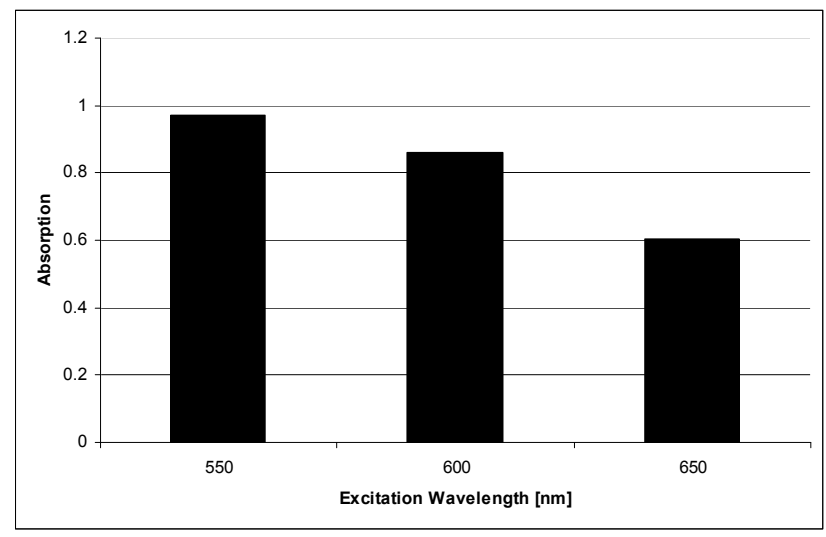

Figure 2. Absorption of photons by blood at 550, 600 and $650 \mathrm{~nm}$

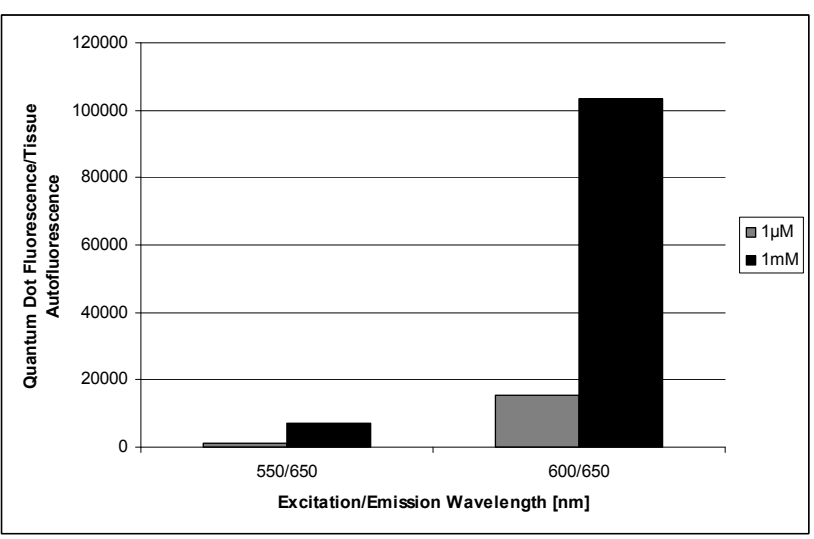

Figure 3. Ratio of quantum dot fluorescence to tissue autofluorescence for $550 / 650 \mathrm{~nm}$ and $600 / 650 \mathrm{~nm}$ excitation/emission wavelengths

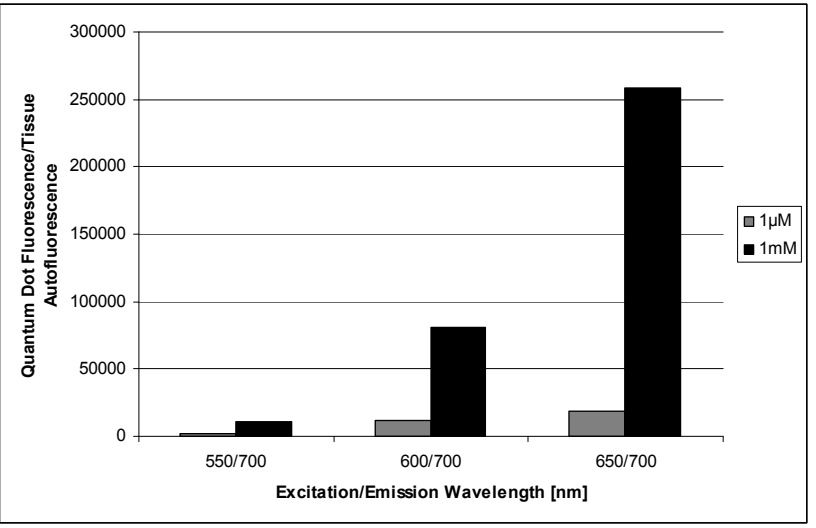

Figure 4. Ratio of quantum dot fluorescence to tissue autofluorescence for $550 / 700 \mathrm{~nm}, 600 / 700 \mathrm{~nm}$ and $650 / 700 \mathrm{~nm}$ excitation/emission wavelengths 


\section{ACKNOWLEDGEMENTS}

The authors thank Thomas Kinter for his technical assistance. Christopher Riederer contributed to this work as a Summer Undergraduate Research Fellow and is currently at Yale University. This work was funded by the Mayo Clinic and Foundation.

\section{REFERENCES}

1. M. Naghavi and e. al. From vulnerable plaque to vulnerable patient. A call for new definitions and risk assessment strategies: Part I. Circulation, 108: 1664-1672, 2003.

2. S.A. Wickline, A.M. Neubauer, P. Winter, S. Caruthers and G. Lanza. Applications of nanotechnology to atherosclerosis, thrombosis and vascular biology. Atherosclerosis, Thrombosis and Vascular Biology, 36: 345-441, 2006.

3. F.A. Jaffer, P. Libby and R. Weissleder. Molecular and cellular imaging of atherosclerosis. Emerging applications. Journal of the American College of Cardiology, 47(7): 1328 - 1338, 2006.

4. W.C.W. Chan and S. Nie. Quantum dot bioconjugates for ultrasensitive nonisotopic detection. Science, 281: 2016-2018, September 1998.

5. M. Bruchez, M. Moronne, P. Gin, S. Weiss and A.P. Alivisatos. Semiconductor nanocrystals as fluorescent biological labels. Science, 281: 2013-2016, September 1998.

6. R. Weissleder and V. Ntziachristos. Shedding light onto live molecular targets. Nature Medicine, 9(1): 123-128, 2003.

7. M.J. McShane, S. Ragestar, M. Pishko and G.L. Cote. Monte Carlo modeling for implantable fluorescent analyte sensors. IEEE Transactions on Biomedical Engineering, 47(5): 624-632, 2000.

8. Q. Liu, C. Zhu and N. Ramanujam. Experimental validation of Monte Carlo modeling of fluorescence in tissues in the UV-visible spectrum. Journal of Biomedical Optics, 8(2): 223-236, 2003.

9. A.J. Welch, C. Gardner, R. Richards-Kortum, E. Chan, G. Criswell, J. Pfefer and S. Warren. Propagation of fluorescent light. Lasers in Surgery and Medicine, 21(166178): 166-178, 1997.

10. L.V. Wang, S.L. Jacques and L. Zheng. MCML Monte Carlo modeling of light transport in multi-layered tissues. Computer Methods and Programs in Biomedicine, 47: 131-146, 1995.

11. M. Keijzer, R. Richards-Kortum, S.L. Jacques and M.S. Feld. Fluorescence spectroscopy of turbid media:

Autofluorescence of the human aorta. Applied Optics, 28(20): 4286-4292, 1989.

12. A. Roggan, M. Friebel, K. Dorschel, A. Hahn and G. Muller. Optical properties of circulating human blood in the wavelength range 400-2500nm. Journal of Biomedical Optics, 4(1): 36-46, 1999. 